\title{
On adjacent vertex distinguishing total coloring of quadrilateral snake
}

\author{
K.Thirusangu ${ }^{1}$ and R.Ezhilarasi ${ }^{2}$ \\ Department of Mathematics, S.I.V.E.T College, Gowrivakkam, Chennai - 600 073, India. \\ Ramanujan Institute for Advanced Study in Mathematics, \\ University of Madras, Chennai - 600005 , India. \\ ${ }^{1}$ kthirusangu@gmail.com, ${ }^{2}$ ezhilarasi@unom.ac.in
}

\section{ABSTRACT}

In this paper, we prove the existence of the adjacent vertex distinguishing total coloring of quadrilateral snake, double quadrilateral snake, alternate quadrilateral snake and double alternate quadrilateral snake in detail. Also, we present an algorithm to obtain the adjacent vertex distinguishing total coloring of these quadrilateral graph family. The minimum number of colors required to give an adjacent vertex distinguishing total coloring (abbreviated as AVDTC) to the graph $G$ is denoted by $\chi_{\text {avt }}(G)$.

Keywords: simple graph; adjacent vertex distinguishing total coloring; adjacent vertex distinguishing total chromatic number; quadrilateral snake; double quadrilateral snake; alternate quadrilateral snake and double alternate quadrilateral snake.

AMS Mathematics Subject Classification : 05C85, 05C15, 05C62, 05C76

\section{Introduction}

In this paper, all graphs are finite, simple and undirected. A graph $G$ consists of a set of vertices $V(G)$ and a set of edges $E(G)$. For every vertex $u, v \in V(G)$, the edge connecting two vertices is denoted by $u v \in E(G)$. The degree of a vertex $v$ of a graph $G$ is denoted by $\operatorname{deg}(v)$. Let $\Delta(G)$ denote the maximum degree of a graph $G$. For standard terminology and concepts of graph theory, we refer [1], [2], [3]. For graphs $G_{1}$ and $G_{2}$, we let $G_{1} \cup G_{2}$ denotes their union, that is, $V\left(G_{1} \cup G_{2}\right)=V\left(G_{1}\right) \cup V\left(G_{2}\right)$ and $E\left(G_{1} \cup G_{2}\right)=E\left(G_{1}\right) \cup E\left(G_{2}\right)$. Let $n$ be any real number. Then $\lfloor n\rfloor$ stands for the largest integer less than or equal to $n$. [6] It has been shown that, if a simple graph $G$ has two adjacent vertices of maximum degree, then $\psi_{\text {avt }}(G) \geq \Delta(G)+2$. Otherwise, if a simple graph $G$ does not have two adjacent vertices of maximum degree, then $\psi_{\text {avt }}(G)=\Delta(G)+1$. The adjacent vertex distinguishing total chromatic number of triangular snake family has been obtained in the literature [4].

\section{Preliminary Results}

In this section, we write some basic definitions and results which are needed for next section.

Definition 2.1 The Quadrilateral snake $Q_{n}$ is obtained from path $P_{n}$ with $\left\{v_{1}, v_{2}, \cdots v_{n}\right\}$ vertices by replacing each edge of the path by $C_{4}$ with a new vertices $\left\{u_{1}, u_{2}, \cdots, u_{n-1}\right\}$ and $\left\{w_{1}, w_{2}, \cdots, w_{n-1}\right\}$.

$$
V\left[Q_{n}\right]=\left\{\left(\bigcup_{i=1}^{n} v_{i}\right) \cup\left(\bigcup_{i=1}^{n-1}\left(u_{i} \cup w_{i}\right)\right)\right\}
$$




$$
E\left[Q_{n}\right]=\left\{\bigcup_{i=1}^{n-1}\left(v_{i} v_{i+1} \cup v_{i} u_{i} \cup v_{i+1} w_{i} \cup u_{i} w_{i}\right)\right\}
$$

Definition 2.2 The Alternate Quadrilateral snake $A\left(Q_{n}\right)$ is obtained from the path $P_{n}$ with every alternate edge of a path is replaced by $C_{4}$. Here, the quadrilateral starts with either the vertex $v_{1}$ or with $v_{2}$.

Definition 2.3 The Double Quadrilateral snake $D\left(Q_{n}\right)$ consists of two quadrilateral snakes that have a common Path using the new vertices $u_{i}, w_{i}, u_{i}^{\prime}$ and $w_{i}^{\prime}$ for $i=1,2, \cdots, n-1$. The vertex set and edge set is given by

$$
\begin{gathered}
V\left[D\left(Q_{n}\right)\right]=\left\{\left(\bigcup_{i=1}^{n} v_{i}\right) \cup\left(\bigcup_{i=1}^{n-1}\left(u_{i} \cup w_{i} \cup u_{i}^{\prime} \cup w_{i}^{\prime}\right)\right)\right\} \\
E\left[D\left(Q_{n}\right)\right]=\left\{\bigcup_{i=1}^{n-1}\left(v_{i} v_{i+1} \cup v_{i} u_{i} \cup v_{i+1} w_{i} \cup u_{i} w_{i} \cup u_{i}^{\prime} w_{i}^{\prime} \cup v_{i} u_{i}^{\prime} \cup v_{i+1} w_{i}^{\prime}\right)\right\}
\end{gathered}
$$

Definition 2.4 The [5] Alternate Double Quadrilateral snake $D A\left(Q_{n}\right)$ consists of two Alternate Quadrilateral snakes that have a common Path.

Definition 2.5 A total $k$-coloring of $G$ is a mapping $f: V(G) \cup E(G) \rightarrow\{1,2, \cdots, k\}, k \in \mathrm{Z}^{+}$such that any two adjacent or incident elements in $V(G) \cup E(G)$ have different colors. A proper total $k$-coloring of $G$ is adjacent vertex distinguishing, if $C_{f}(u) \neq C_{f}(v)$ whenever $u v \in E(G)$, the color set of the vertex $v$ (with respect to $f$ ), we denote $C_{f}(v)$ as $C(v)$.

$$
\begin{array}{rr}
C(v)= & \{\{f(v)\} \cup\{f(v w) \mid v w \in E(G)\}\} \\
\bar{C}(v)= & \{1,2, \cdots, k\} \backslash C(v)
\end{array}
$$

The well-known AVDTC conjecture, made by Zhang et al [6] says that every simple graph $G$ has $\chi_{\text {avt }}(G) \leq \Delta(G)+3$.

\section{AVDTC of $Q_{n}$ and $A\left(Q_{n}\right)$}

In this section, we present an algorithm to obtain the adjacent vertex distinguishing total chromatic number of quadrilateral snake and alternate quadrilateral snake and also we discussed their color classes.

Algorithm 3.1 Procedure: Adjacent vertex distinguishing total coloring of Quadrilateral snake $Q(n)$, for $n \geq 4$.

Input: $G\left(V\left(Q_{n}\right), E\left(Q_{n}\right)\right)$

for $i=1,2, \cdots, n$ do

if $i \equiv 1(\bmod 2)$

$$
f\left(v_{i}\right) \leftarrow 1
$$

else 


$$
f\left(v_{i}\right) \leftarrow 2
$$

end for

for $1 \leq i \leq n-1$ do

$$
f\left(u_{i} w_{i}\right) \leftarrow 2 ; f\left(u_{i}\right) \leftarrow 3 ; f\left(w_{i}\right) \leftarrow 4 ; f\left(u_{i} v_{i}\right) \leftarrow 5 ; f\left(w_{i} v_{i+1}\right) \leftarrow 6
$$

if $i \equiv 1(\bmod 2)$

$$
f\left(v_{i} v_{i+1}\right) \leftarrow 3
$$

else

$$
f\left(v_{i} v_{i+1}\right) \leftarrow 4
$$

end for

end procedure

Output: Adjacent vertex distinguishing total colored of $Q_{n}$, for $n \geq 4$.

Theorem 3.1 The Quadrilateral snake $Q_{n}$ admits $A V D T C$ and

$$
\chi_{a v t}\left(Q_{n}\right)=6, n \geq 4 .
$$

Proof. From the definition (2.1), we have the vertex and edge set of $Q_{n}$.

Therefore $\left|V\left(Q_{n}\right)\right|=3 n-2$ and $\left|E\left(Q_{n}\right)\right|=4(n-1)$.

Also, $\operatorname{deg}\left(v_{1}\right)=2, \operatorname{deg}\left(v_{n}\right)=2, \operatorname{deg}\left(v_{i}\right)=4$ for $2 \leq i \leq n-1$

and $\operatorname{deg}\left(u_{i}\right)=\operatorname{deg}\left(w_{i}\right)=2$ for $i=1$ to $n-1$.

The graph is colored using algorithm (3.1). Now the color classes for $n \geq 4$ is given by

$$
C\left(v_{1}\right)=\{1,3,5\} \text { and } C\left(v_{n}\right)=\left\{\begin{array}{l}
\{2,3,6\}, \text { if } n \equiv 0(\bmod 2) \\
\{1,4,6\}, \text { if } n \equiv 1(\bmod 2)
\end{array}\right.
$$

For $2 \leq i \leq n-1$

$$
\bar{C}\left(v_{i}\right)=\left\{\begin{array}{l}
\{1\}, \text { if } i \equiv 0(\bmod 2) \\
\{2\}, \text { if } i \equiv 1(\bmod 2)
\end{array}\right.
$$

For $1 \leq i \leq n-1$

$$
C\left(u_{i}\right)=\{2,3,5\} \text { and } C\left(w_{i}\right)=\{2,4,6\}
$$

Clearly, the color classes of any two adjacent vertices are different.

$$
\therefore \quad \chi_{\text {avt }}\left(Q_{n}\right)=6, \quad n \geq 4 \text {. }
$$

Algorithm 3.2 Procedure: Adjacent vertex distinguishing total coloring of $A\left(Q_{n}\right)$

for $n \geq 4$. 
Input:

$V\left[A\left(Q_{n}\right)\right] \leftarrow\left\{\begin{array}{cc}\left(\bigcup_{i=1}^{n} v_{i}\right) \cup\left(\bigcup_{i=1}^{\left\lfloor\frac{n}{2}\right\rfloor}\left(u_{i} \cup w_{i}\right)\right), & \text { for } n \equiv 1(\bmod 2) \\ \left(\bigcup_{i=1}^{n} v_{i}\right) \cup\left(\bigcup_{i=1}^{\left(\frac{n}{2}\right)}\left(u_{i} \cup w_{i}\right)\right), & \text { if } \operatorname{deg}\left(v_{1}\right)=\operatorname{deg}\left(v_{n}\right)=2\end{array}\right.$

for $i=1$ to $n$ do

if $i \equiv 1(\bmod 2)$

$$
f\left(v_{i}\right) \leftarrow 1
$$

else

$$
f\left(v_{i}\right) \leftarrow 2
$$

end for

for $i=1$ to $n-1$ do

if $i \equiv 1(\bmod 2)$

$$
f\left(v_{i} v_{i+1}\right) \leftarrow 3
$$

else

$$
f\left(v_{i} v_{i+1}\right) \leftarrow 4
$$

end for

if $n \equiv 1(\bmod 2)$

$$
\begin{aligned}
& \text { for } i=1 \text { to }\left\lfloor\frac{n}{2}\right\rfloor \text { do } \\
& \quad f\left(u_{i}\right) \leftarrow 3 ; f\left(w_{i}\right) \leftarrow 4 ; f\left(u_{i} w_{i}\right) \leftarrow 2 \\
& \text { if } \operatorname{deg}\left(v_{n}\right)=1 \\
& \qquad f\left(v_{2 i-1} u_{i}\right)=f\left(v_{2 i} w_{i}\right) \leftarrow 5 \\
& \text { else } \\
& \qquad f\left(v_{2 i+1} w_{i}\right)=f\left(v_{2 i} u_{i}\right) \leftarrow 5 \\
& \text { end for }
\end{aligned}
$$


else

if $\operatorname{deg}\left(v_{n}\right)=\operatorname{deg}\left(v_{1}\right)=2$

$$
\begin{aligned}
& \text { for } i=1 \text { to }\left(\frac{n}{2}\right) \text { do } \\
& \qquad f\left(u_{i}\right) \leftarrow 3 ; f\left(w_{i}\right) \leftarrow 4 ; f\left(u_{i} w_{i}\right) \leftarrow 2 ; f\left(v_{2 i-1} u_{i}\right)=f\left(v_{2 i} w_{i}\right) \leftarrow 5
\end{aligned}
$$

end for

else

$$
\begin{aligned}
& \text { for } i=1 \text { to }\left\lfloor\frac{n-1}{2}\right\rfloor \text { do } \\
& \qquad f\left(u_{i}\right) \leftarrow 3 ; f\left(w_{i}\right) \leftarrow 4 ; f\left(u_{i} w_{i}\right) \leftarrow 2 ; f\left(v_{2 i+1} w_{i}\right)=f\left(v_{2 i} u_{i}\right) \leftarrow 5
\end{aligned}
$$

end for

\section{end procedure}

Output: Adjacent vertex distinguishing total colored of $A\left(Q_{n}\right)$, for $n \geq 4$.

Theorem 3.2 The Alternate Quadrilateral snake $A\left(Q_{n}\right)$ admits $A V D T C$ and

$$
\chi_{\text {avt }}\left(A\left(Q_{n}\right)\right)=5, \quad \text { for } n \geq 4 \text {. }
$$

Proof. The vertex set of Alternate Quadrilateral snake $A\left(Q_{n}\right)$ is given in the algorithm (3.2). Here we have two cases for $n$ is even or odd. In each case, the quadrilateral starts with either the vertex of the path $v_{1}$ or $v_{2}$.

Case-1. When $n \equiv 1(\bmod 2)$.

Suppose the quadrilateral starts with $v_{1}$, then $\operatorname{deg}\left(v_{n}\right)=1$ and if the quadrilateral starts with $v_{2}$, then $\operatorname{deg}\left(v_{1}\right)=1$. The edge set of $A\left(Q_{n}\right)$ is given by

$$
E\left(A\left(Q_{n}\right)\right)=\left\{\begin{array}{l}
\left(\bigcup_{i=1}^{n-1} v_{i} v_{i+1}\right) \cup\left(\bigcup_{i=1}^{\left\lfloor\frac{n}{2}\right\rfloor}\left(\left(v_{2 i-1} u_{i}\right) \cup\left(v_{2 i} w_{i}\right) \cup\left(u_{i} w_{i}\right)\right)\right), \text { if } \operatorname{deg}\left(v_{n}\right)=1 \\
\left(\bigcup_{i=1}^{n-1} v_{i} v_{i+1}\right) \cup\left(\bigcup_{i=1}^{\left(\frac{n}{2}\right\rfloor}\left(\left(v_{2 i+1} w_{i}\right) \cup\left(v_{2 i} u_{i}\right) \cup\left(u_{i} w_{i}\right)\right)\right), \text { if } \operatorname{deg}\left(v_{1}\right)=1
\end{array}\right.
$$

The color classes of $A\left(Q_{n}\right)$ is given by

For $2 \leq i \leq n-1$ 


$$
\bar{C}\left(v_{i}\right)=\left\{\begin{array}{l}
\{2\}, \text { if } i \equiv 1(\bmod 2) \\
\{1\}, \text { if } i \equiv 0(\bmod 2)
\end{array}\right.
$$

For $i=1,2, \cdots,\left\lfloor\frac{n}{2}\right\rfloor$

$$
C\left(u_{i}\right)=\{2,3,5\} \text { and } C\left(w_{i}\right)=\{2,4,5\}
$$

If $\operatorname{deg}\left(v_{n}\right)=1$ then

$$
C\left(v_{1}\right)=\{1,3,5\} \text { and } C\left(v_{n}\right)=\{1,4\}
$$

If $\operatorname{deg}\left(v_{1}\right)=1$, then

$$
C\left(v_{1}\right)=\{1,3\} \text { and } C\left(v_{n}\right)=\{1,4,5\}
$$

Case-2. When $n \equiv 0(\bmod 2)$.

Here, we have the two cases that the quadrilatral starts with $v_{1}$, then $\operatorname{deg}\left(v_{1}, v_{n}\right)=2$ and starts with $v_{2}$, then $\operatorname{deg}\left(v_{1}, v_{n}\right)=1$.

$E\left(A\left(Q_{n}\right)\right)=\left\{\begin{array}{l}\left(\bigcup_{i=1}^{n-1} v_{i} v_{i+1}\right) \cup\left(\bigcup_{i=1}^{\left.\frac{n}{2}\right)}\left(\left(v_{2 i-1} u_{i}\right) \cup\left(v_{2 i} w_{i}\right) \cup\left(u_{i} w_{i}\right)\right)\right), \text { if } \operatorname{deg}\left(v_{1}\right)=\operatorname{deg}\left(v_{n}\right)=2 \\ \left(\bigcup_{i=1}^{n-1} v_{i} v_{i+1}\right) \cup\left(\bigcup_{i=1}^{\left\lfloor\frac{n-1}{2}\right\rfloor}\left(\left(v_{2 i+1} w_{i}\right) \cup\left(v_{2 i} u_{i}\right) \cup\left(u_{i} w_{i}\right)\right)\right), \text { if } \operatorname{deg}\left(v_{1}\right)=\operatorname{deg}\left(v_{n}\right)=1\end{array}\right.$

If $\operatorname{deg}\left(v_{1}\right)=\operatorname{deg}\left(v_{n}\right)=2$, then

$$
\left|V\left(A\left(Q_{n}\right)\right)\right|=2 n \text { and }\left|E\left(A\left(Q_{n}\right)\right)\right|=\frac{5 n-2}{2}
$$

The color classes of $A\left(Q_{n}\right)$ is given by

$$
\text { For } 1 \leq i \leq\left(\frac{n}{2}\right)
$$

$$
C\left(u_{i}\right)=\{2,3,5\} \text { and } C\left(w_{i}\right)=\{2,4,5\}
$$

For $2 \leq i \leq n-1$

$$
\begin{gathered}
\bar{C}\left(v_{i}\right)=\left\{\begin{array}{l}
\{2\}, \text { if } i \equiv 1(\bmod 2) \\
\{1\}, \text { if } i \equiv 0(\bmod 2)
\end{array}\right. \\
C\left(v_{1}\right)=\{1,3,5\} \text { and } C\left(v_{n}\right)=\{2,3,5\}
\end{gathered}
$$


If $\operatorname{deg}\left(v_{1}\right)=\operatorname{deg}\left(v_{n}\right)=1$, then

$$
\left|V\left(A\left(Q_{n}\right)\right)\right|=2 n-2 \text { and }\left|E\left(A\left(Q_{n}\right)\right)\right|=\frac{5 n-8}{2}
$$

The color classes are for $i=1,2, \cdots\left\lfloor\frac{n-1}{2}\right\rfloor$

$$
C\left(v_{1}\right)=\{1,3\}, C\left(v_{n}\right)=\{2,3\}, C\left(u_{i}\right)=\{2,3,5\} \text { and } C\left(w_{i}\right)=\{2,4,5\}
$$

For $2 \leq i \leq n-1$

$$
\bar{C}\left(v_{i}\right)=\left\{\begin{array}{l}
\{1\}, \text { if } i \equiv 0(\bmod 2) \\
\{2\}, \text { if } i \equiv 1(\bmod 2)
\end{array}\right.
$$

Clearly, the color classes of any two adjacent vertices are different.

$$
\therefore \chi_{\text {avt }}\left(A\left(Q_{n}\right)\right)=5 \text {, for } n \geq 4 \text {. }
$$

\section{AVDTC of $D\left(Q_{n}\right)$ and $D A\left(Q_{n}\right)$}

In this section, we present an algorithm to obtain the adjacent vertex distinguishing total chromatic number of Double Quadrilateral snake and Double Alternate Quadrilateral snake and also we discussed their color classes in detail.

Algorithm 4.1 Procedure: Adjacent vertex distinguishing total coloring of Double Quadrilateral snake $D\left(Q_{n}\right)$, for $n \geq 4$.

Input: $G\left(V\left(D\left(Q_{n}\right)\right), E\left(D\left(Q_{n}\right)\right)\right)$

$$
\begin{aligned}
& \text { for } i=1,2, \cdots, n \text { do } \\
& \text { if } i \equiv 1(\bmod 2) \\
& \quad f\left(v_{i}\right) \leftarrow 1 \\
& \text { else } \\
& \qquad f\left(v_{i}\right) \leftarrow 2 \\
& \text { end for } \\
& \text { for } 1 \leq i \leq n-1 \text { do } \\
& \quad f\left(u_{i} w_{i}\right)=f\left(u_{i}^{\prime} w_{i}^{\prime}\right) \leftarrow 2 ; f\left(u_{i}\right)=f\left(u_{i}^{\prime}\right) \leftarrow 3 ; f\left(w_{i}\right)=f\left(w_{i}^{\prime}\right) \leftarrow 4 \\
& \quad f\left(u_{i} v_{i}\right) \leftarrow 5 ; f\left(u_{i}^{\prime} v_{i}\right) \leftarrow 7 ; f\left(w_{i} v_{i+1}\right) \leftarrow 6 ; f\left(w_{i}^{\prime} v_{i+1}\right) \leftarrow 8 \\
& \text { if } i \equiv 1(\bmod 2) \\
& \qquad f\left(v_{i} v_{i+1}\right) \leftarrow 3 \\
& \quad \text { else } \\
& \qquad f\left(v_{i} v_{i+1}\right) \leftarrow 4 \\
& \text { end for }
\end{aligned}
$$




\section{end procedure}

Output: Adjacent vertex distinguishing total colored of $D\left(Q_{n}\right)$, for $n \geq 4$.

Theorem 4.1 The Double Quadrilateral snake $D\left(Q_{n}\right)$ admits $A V D T C$ and

$$
\chi_{\text {avt }}\left(D\left(Q_{n}\right)\right)=8, n \geq 4 .
$$

Proof. From the definition (2.3), We have the vertex set and edge set of $D\left(Q_{n}\right)$. Therefore $\left|V\left(D\left(Q_{n}\right)\right)\right|=5 n-4$ and $\left|E\left(D\left(Q_{n}\right)\right)\right|=7(n-1)$. Also, $\operatorname{deg}\left(v_{1}\right)=\operatorname{deg}\left(v_{n}\right)=3, \operatorname{deg}\left(v_{i}\right)=6$ for $2 \leq i \leq n-1$ and $\operatorname{deg}\left(u_{i}\right)=\operatorname{deg}\left(u_{i}^{\prime}\right)=\operatorname{deg}\left(w_{i}\right)=\operatorname{deg}\left(w_{i}^{\prime}\right)=2$ for $i=1$ to $n-1$. We have colored the graph using algorithm (4.1), for $n \geq 4$. Now the color classes are given by

$$
C\left(v_{1}\right)=\{1,3,5,7\} \text { and } C\left(v_{n}\right)=\left\{\begin{array}{l}
\{2,3,6,8\}, \text { if } n \equiv 0(\bmod 2) \\
\{1,4,6,8\}, \text { if } n \equiv 1(\bmod 2)
\end{array}\right.
$$

For $2 \leq i \leq n-1$

$$
\bar{C}\left(v_{i}\right)=\left\{\begin{array}{l}
\{1\}, \text { if } i \equiv 0(\bmod 2) \\
\{2\}, \text { if } i \equiv 1(\bmod 2)
\end{array}\right.
$$

For $1 \leq i \leq n-1$

$$
C\left(u_{i}\right)=\{2,3,5\}, C\left(u_{i}^{\prime}\right)=\{2,3,7\}, C\left(w_{i}\right)=\{2,4,6\}, C\left(w_{i}^{\prime}\right)=\{2,4,8\}
$$

Clearly, the color set of any two adjacent vertices are different.

$$
\therefore \quad \chi_{\text {avt }}\left(D\left(Q_{n}\right)\right)=8, \quad n \geq 4 \text {. }
$$

Algorithm 4.2 Procedure: Adjacent vertex distinguishing total coloring of

$D A\left(Q_{n}\right)$ for $n \geq 4$.

\section{Input:}

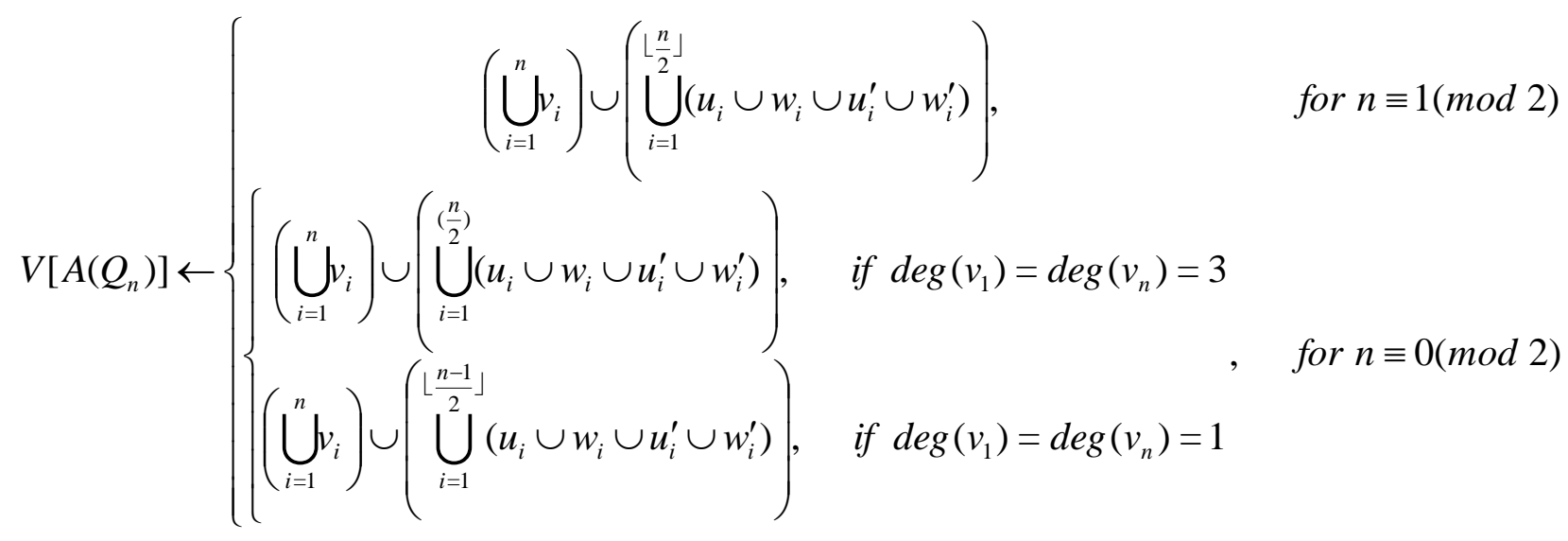

for $i=1$ to $n$ do 
if $i \equiv 1(\bmod 2)$

$f\left(v_{i}\right) \leftarrow 1$

else

$f\left(v_{i}\right) \leftarrow 2$

end for

for $i=1$ to $n-1$ do

if $i \equiv 1(\bmod 2)$

$f\left(v_{i} v_{i+1}\right) \leftarrow 3$

else

$f\left(v_{i} v_{i+1}\right) \leftarrow 4$

end for

if $n \equiv 1(\bmod 2)$

$$
\begin{aligned}
& \text { for } i=1 \text { to }\left\lfloor\frac{n}{2}\right\rfloor \text { do } \\
& f\left(u_{i}\right)=f\left(u_{i}^{\prime}\right) \leftarrow 3 ; f\left(w_{i}\right)=f\left(w_{i}^{\prime}\right) \leftarrow 4 ; f\left(u_{i} w_{i}\right)=f\left(u_{i}^{\prime} w_{i}^{\prime}\right) \leftarrow 2 \\
& \text { if } \operatorname{deg}\left(v_{n}\right)=1 \\
& \quad f\left(v_{2 i-1} u_{i}\right)=f\left(v_{2 i} w_{i}\right) \leftarrow 5 ; f\left(v_{2 i-1} u_{i}^{\prime}\right)=f\left(v_{2 i} w_{i}^{\prime}\right) \leftarrow 6
\end{aligned}
$$

else

$$
f\left(v_{2 i+1} u_{i}\right)=f\left(v_{2 i} w_{i}\right) \leftarrow 5 ; f\left(v_{2 i+1} u_{i}^{\prime}\right)=f\left(v_{2 i} w_{i}^{\prime}\right) \leftarrow 6
$$

end for

else

if $\operatorname{deg}\left(v_{n}\right)=\operatorname{deg}\left(v_{1}\right)=3$

$$
\begin{aligned}
& \text { for } i=1 \text { to } \frac{n}{2} \text { do } \\
& f\left(u_{i}\right)=f\left(u_{i}^{\prime}\right) \leftarrow 3 ; f\left(w_{i}\right)=f\left(w_{i}^{\prime}\right) \leftarrow 4 ; f\left(u_{i} w_{i}\right)=f\left(u_{i}^{\prime} w_{i}^{\prime}\right) \leftarrow 2 \\
& f\left(v_{2 i-1} u_{i}\right)=f\left(v_{2 i} w_{i}\right) \leftarrow 5 ; f\left(v_{2 i-1} u_{i}^{\prime}\right)=f\left(v_{2 i} w_{i}^{\prime}\right) \leftarrow 6
\end{aligned}
$$

end for

else

$$
\text { for } i=1 \text { to }\left\lfloor\frac{n-1}{2}\right\rfloor \text { do }
$$




$$
\begin{aligned}
& f\left(u_{i}\right)=f\left(u_{i}^{\prime}\right) \leftarrow 3 ; f\left(w_{i}\right)=f\left(w_{i}^{\prime}\right) \leftarrow 4 ; f\left(u_{i} w_{i}\right)=f\left(u_{i}^{\prime} w_{i}^{\prime}\right) \leftarrow 2 \\
& f\left(v_{2 i+1} w_{i}\right)=f\left(v_{2 i} u_{i}\right) \leftarrow 5 ; f\left(v_{2 i+1} w_{i}^{\prime}\right)=f\left(v_{2 i} u_{i}^{\prime}\right) \leftarrow 6
\end{aligned}
$$

end for

\section{end procedure}

Output: Adjacent vertex distinguishing total colored of $D A\left(Q_{n}\right)$, for $n \geq 4$.

Theorem 4.2 The Double Alternate Quadrilateral snake $D A\left(Q_{n}\right)$ admits $A V D T C$

$$
\text { and } \chi_{\text {avt }}\left(D A\left(Q_{n}\right)\right)=6, \text { for } n \geq 4 \text {. }
$$

Proof. The vertex set of Double Alternate Quadrilateral snake is given in the algorithm (4.2). Here we discussed two cases that when $n$ is even or odd. In each case, the quadrilateral starts with either the vertex $v_{1}$ or with $v_{2}$.

Case-1. When $n \equiv 1(\bmod 2)$.

$$
\begin{aligned}
& E\left[D A\left(Q_{n}\right)\right]=\left\{\begin{array}{l}
\left(\bigcup_{i=1}^{n-1} v_{i} v_{i+1}\right) \cup\left(\bigcup_{i=1}^{\left\lfloor\frac{n}{2}\right\rfloor}\left(v_{2 i-1} u_{i} \cup v_{2 i} w_{i} \cup u_{i} w_{i} \cup v_{2 i-1} u_{i}^{\prime} \cup v_{2 i} w_{i}^{\prime} \cup u_{i}^{\prime} w_{i}^{\prime}\right)\right), \text { if } \operatorname{deg}\left(v_{n}\right)=1 \\
\left(\bigcup_{i=1}^{n-1} v_{i} v_{i+1}\right) \cup\left(\bigcup_{i=1}^{\left\lfloor\frac{n}{2}\right\rfloor}\left(v_{2 i+1} w_{i} \cup v_{2 i} u_{i} \cup u_{i} w_{i} \cup v_{2 i+1} w_{i}^{\prime} \cup v_{2 i} u_{i}^{\prime} \cup u_{i}^{\prime} w_{i}^{\prime}\right)\right), \text { if } \operatorname{deg}\left(v_{1}\right)=1
\end{array}\right. \\
& \left|V\left(D A\left(Q_{n}\right)\right)\right|=3 n-2 \text { and }\left|E\left(D A\left(Q_{n}\right)\right)\right|=4 n-4
\end{aligned}
$$

The color classes of $D A\left(Q_{n}\right)$ for $n \geq 4$ is given by

For $2 \leq i \leq n-1$

$$
\bar{C}\left(v_{i}\right)=\left\{\begin{array}{l}
\{2\}, \text { if } i \equiv 1(\bmod 2) \\
\{1\}, \text { if } i \equiv 0(\bmod 2)
\end{array}\right.
$$

For $i=1,2, \cdots,\left\lfloor\frac{n}{2}\right\rfloor$

$$
\begin{aligned}
& C\left(u_{i}\right)=\{2,3,5\} \text { and } C\left(w_{i}\right)=\{2,4,5\} \\
& C\left(u_{i}^{\prime}\right)=\{2,3,6\} \text { and } C\left(w_{i}^{\prime}\right)=\{2,4,6\}
\end{aligned}
$$

If $\operatorname{deg}\left(v_{n}\right)=1$ then

$$
C\left(v_{1}\right)=\{1,3,5,6\} \text { and } C\left(v_{n}\right)=\{1,4\}
$$

If $\operatorname{deg}\left(v_{1}\right)=1$, then

$$
C\left(v_{1}\right)=\{1,3\} \text { and } C\left(v_{n}\right)=\{1,4,5,6\}
$$

Case-2. When $n \equiv 0(\bmod 2)$. 


$$
E\left(D A\left(Q_{n}\right)\right)=\left\{\begin{array}{l}
\left(\bigcup_{i=1}^{n-1} v_{i} v_{i+1}\right) \cup\left(\bigcup_{i=1}^{\left(\frac{n}{2}\right)}\left(v_{2 i-1} u_{i} \cup v_{2 i} w_{i} \cup v_{2 i-1} u_{i}^{\prime} \cup v_{2 i} w_{i}^{\prime} \cup u_{i} w_{i} \cup u_{i}^{\prime} w_{i}^{\prime}\right)\right), \text { if } \operatorname{deg}\left(v_{1}\right)=\operatorname{deg}\left(v_{n}\right)=3 \\
\left(\bigcup_{i=1}^{n-1} v_{i} v_{i+1}\right) \cup\left(\bigcup_{i=1}^{\left\lfloor\frac{n-1}{2}\right\rfloor}\left(v_{2 i+1} w_{i} \cup v_{2 i} u_{i} \cup v_{2 i+1} w_{i}^{\prime} \cup v_{2 i} u_{i}^{\prime} \cup u_{i} w_{i} \cup u_{i}^{\prime} w_{i}^{\prime}\right)\right), \text { if } \operatorname{deg}\left(v_{1}\right)=\operatorname{deg}\left(v_{n}\right)=1
\end{array}\right.
$$

If $\operatorname{deg}\left(v_{1}\right)=\operatorname{deg}\left(v_{n}\right)=3$, then

$$
\left|V\left(D A\left(Q_{n}\right)\right)\right|=3 n \text { and }\left|E\left(D A\left(Q_{n}\right)\right)\right|=4 n-1
$$

The color classes of $D A\left(Q_{n}\right)$ for $n \geq 4$ is given by

$$
\begin{aligned}
& \text { For } 1 \leq i \leq\left(\frac{n}{2}\right) \\
& \qquad C\left(u_{i}\right)=\{2,3,5\}, C\left(w_{i}\right)=\{2,4,5\}, C\left(u_{i}^{\prime}\right)=\{2,3,6\}, C\left(w_{i}^{\prime}\right)=\{2,4,6\}
\end{aligned}
$$

For $2 \leq i \leq n-1$

$$
\begin{gathered}
\bar{C}\left(v_{i}\right)=\left\{\begin{array}{l}
\{2\}, \text { if } i \equiv 1(\bmod 2) \\
\{1\}, \text { if } i \equiv 0(\bmod 2)
\end{array}\right. \\
C\left(v_{1}\right)=\{1,3,5,6\} \text { and } C\left(v_{n}\right)=\{2,3,5,6\}
\end{gathered}
$$

If $\operatorname{deg}\left(v_{1}\right)=1$ and $\operatorname{deg}\left(v_{n}\right)=1$, then

$$
\left|V\left(D A\left(Q_{n}\right)\right)\right|=3 n-4 \text { and }\left|E\left(D A\left(Q_{n}\right)\right)\right|=4 n-7
$$

The color classes are

$$
C\left(v_{1}\right)=\{1,3\} \text { and } C\left(v_{n}\right)=\{2,3\}
$$

for $i=1,2, \cdots\left\lfloor\frac{n-1}{2}\right\rfloor$

$$
\begin{aligned}
& C\left(u_{i}\right)=\{2,3,5\} \text { and } C\left(w_{i}\right)=\{2,4,5\} \\
& C\left(u_{i}^{\prime}\right)=\{2,3,6\} \text { and } C\left(w_{i}^{\prime}\right)=\{2,4,6\}
\end{aligned}
$$

For $2 \leq i \leq n-1$

$$
\bar{C}\left(v_{i}\right)=\left\{\begin{array}{l}
\{1\}, \text { if } i \equiv 0(\bmod 2) \\
\{2\}, \text { if } i \equiv 1(\bmod 2)
\end{array}\right.
$$

Clearly, the color classes of any two adjacent vertices are different.

$$
\therefore \chi_{\text {avt }}\left(D A\left(Q_{n}\right)\right)=6 \text {, for } n \geq 4 \text {. }
$$




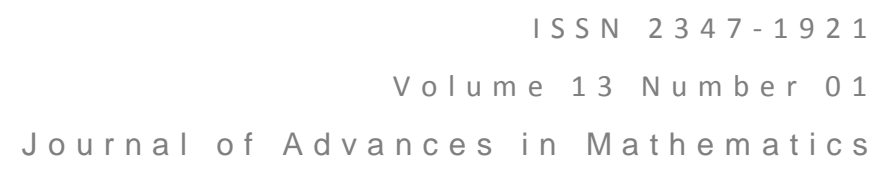

\section{Conclusion.}

We found the adjacent vertex distinguishing total chromatic number of Quadrilateral snake family.

\section{References}

[1] N.L. Biggs, Algebraic Graph Theory, Cambridge University Press, $2^{\text {nd }}$ edition, Cambridge, 1993.

[2] Harary, F., Graph Theory (Addison-Wesley, Reading, Mass. 1969).

[3] K.K.Parthasarathy, Basic Graph Theory, Tata McGraw Hill, 1994.

[4] K.Thirusangu and R.Ezhilarasi "On Adjacent Vertex Distinguishing total coloring of Triangular snake", (communicated).

[5] S.K. Vaidya and N. B. Vyas, "Product cordial labeling for alternate snake graphs", Malaya J. Mat. 2(3)(2014) 188196.

[6] ZHANG Zhongfu, CHEN Xiang 'en, L. Jingwen, YAO Bing, LU Xinzhong and WANG Jianfang. "On AdjacentVertex- Distinguishing total coloring of graphs", Science in China Ser.A Mathematics 2005, Vol. 48 (No.3), 289-299. 